International Journal of Innovative Studies in Aquatic Biology and Fisheries

Volume 5, Issue 4, 2019, PP 16-20

ISSN 2454-7662 (Print) \& ISSN 2454-7670 (Online)

DOI: http://dx.doi.org/10.20431/2454-7670.0504004

www.arcjournals.org

\title{
Research on the Application of Fishery Anesthetic MS-222 and Eugenol in China
}

\author{
Xiao-jiang Chen* \\ Jiangsu Agri-animal Husbandry Vocational College
}

*Corresponding Author: Xiao-jiang Chen, Jiangsu Agri-animal Husbandry Vocational College.

\begin{abstract}
In order to clarify the research and application status of narcotic MS-222 and eugenol on aquatic animals, this article introduces the effects of anesthetic MS-222 and eugenol on oxygen consumption and ammonia excretion rates of different aquatic animals, the changes in resuscitation time and biochemical parameters, the safety of anesthetics and tissue residues after use of anesthetics, and points out that safety should be considered in the selection of anesthetics and the effect of anesthesia is second, and the development prospect of high-efficiency and environmental-friendly anesthetics is broad.
\end{abstract}

Keywords: Fishery anesthetic;MS-222; Eugenol; Oxygen Consumption Rate; Ammonia Excretion Rate

\section{INTRODUCTION}

As a large aquaculture industry country, China has been producing the world's largest number of aquaculture production for many years. In the operation to aquatic animals such as fishing, transportation, artificial propagation, individual marking, internal anatomy and external morphological measurement, etc., aquatic animals will produce strong stress reactions, such as mucus secretion, jumping and struggling, which seriously hamper the smooth development of related work and cause some harm to fish. The scientific and rational use of anesthetics can significantly reduce the stress response of fish, reduce the damage to fish, and facilitate manual operation.

At present, there are many kinds of anesthetics for aquatic animals. There are more than 30 kinds, such as eugenol, MS-222, benzocaine, 2-phenoxyethanol, etc. Among them, MS-222 and eugenol are the most widely used in breeding and production. MS-222 has been approved by the Food and Drug Administration (FDA) of the United States for its advantages of safe and convenient use, fast anaesthesia and recovery. It is the most widely used, safe and effective aquatic anaesthetic at present. MS-222 has been reported as an anesthetic for many kinds of fish [1-4]. Eugenol is a plant extract. It is cheap and easy to get, and the metabolites can be quickly discharged. Eugenol will not induce mutations in the body. It does not cause harm to human beings and the environment. It can be added directly in food. Research of eugenol, as a fish anesthetic of aquaculture animals such as IetalurusPunetaus, has been reported [5-6]. The writer summarizes the anesthetic MS222 and eugenol from the principles of anesthesia and its influencing factors and the current application in production.

\section{ANESTHETIC PRINCIPLES AND INFLUENCING FACTORS OF FISHERY ANESTHETICS}

\subsection{Anesthetic Principles}

The action principle of anesthetics: anesthetics first inhibit the cerebral cortex (tactile loss period), then act on the basal ganglia and cerebellum (excitatory period), and finally act on the spinal cord (anaesthesia period). Too much or too long exposure can make the anesthetics deep into the medulla, causing respiration and blood vessel shrink, and central paralysis, and eventually lead to death [7].

\subsection{Influence Factor}

\subsubsection{Fish Species, Specifications and Ages}

Different breeds react differently to different anesthetics and concentrations, while MS-222 has no effect on the crayfish (Procambarusclarkii). Generally, for the same variety with different specifications, the 
higher the quality is, the more the anesthetic dose is need. As for the same kind and different ages of fish, because of metabolic reasons, respiratory organs are different, the effective anesthetic dose also has obvious changes. In the juvenile stage, skin breathing is the main method, while in the adult stage, gill breathing is the main method. As the age increases, the tolerance to anesthetics will lead to different effective anesthetic doses [8].

\subsubsection{Temperature}

In the aquatic environment where fish grow, when the water temperature rises but the concentration of fishery anesthetics is the same, the time for the receptor to reach anesthesia stage becomes shorter. When the water temperature rises to a certain temperature, the anesthetic effect of some anesthetics will decrease. In most cases, the survival rate of recipients after anesthesia is greatly reduced at higher temperatures.

\subsection{3.pH Value and Salinity}

The change of $\mathrm{pH}$ value has different effects on fish body because of different doses. Generally, it has little effect on freshwater fish and great influence on seawater fish. When high-dose MS-222 is used, a certain amount of $\mathrm{NaHCO} 3$ should be added to buffer it so that the $\mathrm{pH}$ value is between 7.0 and 7.5 and reduce the stimulation to the fish [9].

At high salinity, in order to regulate the balance of osmotic pressure, fish need to consume more energy, and their respiratory rate is accelerated, and their oxygen consumption is increased. Therefore, at high salinity, fish consume more anesthetics, which shorten the time of entering anesthesia, prolong the recovery time and increase the mortality rate.

\subsubsection{Dissolved Oxygen}

The level of dissolved oxygen has a profound effect on the respiration of fish. When dissolved oxygen is low, fish anesthetics play a faster role, but at the same time, recovery time is slower. When dissolved oxygen is high, the receptor is more sensitive to fishery anesthetics, and the dissolved oxygen the receptor needs is low, and the coming of anaesthesia stage of the receptor is slower, but the recovery time is shorter and the survival rate is higher.

\section{STUDY ON THE APPLICATION OF MS-222 IN AQUATIC ANIMALS}

After MS-222 anesthetize aquatic animals, it mainly concentrates in organs such as spleen and liver, and its content in muscle tissue is very small. In clean water environment, it is easy to transfer from aquatic animals to water. Therefore, the anesthetic effect of MS-222 is fast, the recovery time of the receptor is short, the safety is high, and it is harmless to the recipient and the human body contacted. But MS-222 also has its shortcomings. When MS-222 is used during transportation, the CO2 emission cannot be reduced, and the water solution is weak acidic, which lead to the increase of cortisol in plasma after deep anaesthesia. In addition, when we use MS-222 solution, direct sunlight should be avoided, otherwise it will produce strong toxicity to fish in sea water.

\subsection{Anesthesia Effect of MS-222}

The most suitable concentration of MS-222 for bass is $20 \sim 50 \mathrm{mg} / \mathrm{Lunder}$ the condition of $25^{\circ} \mathrm{C}$.After 24 hours of anesthesia, the recovery rate could reach $100 \%$ in clear water environment[10]. The effective concentration of MS-222 for Acipenserschrenckii was $80-100 \mathrm{mg} / \mathrm{L}$ under the condition of $16 \sim 18^{\circ} \mathrm{C}$, which was the safest, and the fish could recover within 3-5 minutes when held in hand[11]. MS-222 had a good anesthetic effect on Pelteobagrusfulvidraco at 40-80 mg/L, and the most suitable concentration for short-time anesthesia was $60 \mathrm{mg} / \mathrm{L}$ [12]. Yan found that, when the concentration of MS-222 was lower than $40 \mathrm{mg} / \mathrm{L}$, it had no anesthetic effect on the Coiliamacrognathos Bleeker, but had a certain sedative effect[13]; Sun showed that, the safest concentration of MS-222 was $60 \mathrm{mg} / \mathrm{L}$ in the transport of cynoglossussemilaevis[14]; Ding found that, the reasonable range of MS-222 anesthesia for bream was between 60 and $80 \mathrm{mg} / \mathrm{L}[2]$; $\mathrm{Xu}$ found that MS-222 had no anesthetic effect on crayfish (Procambarusclarkii). The anesthetic effect of the same anesthetic for different aquatic products is different. Therefore, it is the research direction to continue to explore more anaesthesia mechanisms and 
metabolic pathways of aquatic animals.

\subsection{Effects of MS-222 on Oxygen Consumption Rate and Ammonia Excretion Rate of Aquatic Animals}

Zhang studied the anesthesia of juvenile Oreochromis niloticus. When the concentration of MS-222 was $10 \mathrm{mg} / \mathrm{L}, 20 \mathrm{mg} / \mathrm{L}$ and $30 \mathrm{mg} / \mathrm{L}$, the average oxygen consumption rate decreased by $10 \%, 31.4 \%, 40.5 \%$, and the average ammonia excretion rate decreased by $12.4 \%, 37.8 \%, 42.8 \%[15]$. When the concentration of MS-222 was 20-100 mg/L, the oxygen consumption rate of Procambarus clarkia could not be reduced, but decreased when the concentration exceeded $100 \mathrm{mg} / \mathrm{L}$. The general trend was that with the increase of the concentration, the oxygen consumption rate and ammonia excretion rate increased first and then decreased.

\subsection{Changes of Biochemical Parameters}

Wang found that the serum cortisol content of bass was significantly increased at $25^{\circ} \mathrm{C}$ after bass was anesthetized and transported, and the activity of serum AST increased slightly. After 10h, serum UREA level increased significantly, serum GLU increased significantly, and the activity of serum LDH increased significantly during anesthesia [10].

\subsection{Tissue Residues and Safety Of MS-222}

Xue determined the MS-222 residues in anaesthetized fish. It was found that the half-life period of elimination in muscle and liver were 5.54-5.27h $(30 \mathrm{mg} / \mathrm{L})$ and $8.72-7.15 \mathrm{~h}(60 \mathrm{mg} / \mathrm{L})$ respectively [16]. When the concentration was $30 \mathrm{mg} / \mathrm{L}$, the withdrawal period was at least $4.5 \mathrm{~d}$; if the concentration was 60 $\mathrm{mg} / \mathrm{L}$, the withdrawal period was at least 7.5day. With the deepening of research and the emphasis on food safety, many developed countries have strictly stipulated MS-222 withdrawal period. For example, the United States stipulated that MS-222 withdrawal period is 21day, Canada is 5day, New Zealand is 10day [17]. Sun suggested that the withdrawal period of cynoglossussemilaevis after being anaesthetized by MS-222 should not be less than 16day [14].

\section{RESEARCH AND EXPERIMENT OF EUGENOL IN AQUATIC ANIMALS}

Eugenol, its molecular formula $\mathrm{C} 10 \mathrm{H} 12 \mathrm{O} 2$, is a colorless to yellowish slightly-thickened liquid. If exposed to air for a long time, it will turn into a black-brown liquid. Eugenol and its metabolites can be quickly discharged from blood and tissues, and it will not induce mutant substances in the body. Eugenol has the advantages of high efficiency and low dose. Compared with MS-222, its recovery time is longer. Owing to its volatility, its effect will gradually decrease during anesthesia.

\subsection{Anesthetic Effect of Eugenol}

The anesthetic effect of clove oil on loach was the most suitable when it was at $60-100 \mathrm{mg} / \mathrm{L}$, and it would not cause harm to loach in breeding production and scientific research [18]. Kuang showed that when the concentration of eugenol anesthetics was not higher than $27 \mathrm{mg} / \mathrm{L}$, the anesthetic effect on ictalurus punctatus was the safest [19]; when the concentration of eugenol anesthetics was $5 \mathrm{mg} / \mathrm{L}$ and 8 $\mathrm{mg} / \mathrm{L}$, it would not produce anesthetic effect on Coiliaectenes within 12 hours[13]. $40 \mathrm{mg} / \mathrm{L}$ eugenol can produce anesthetic effect onprocambarusclarkii.

\subsection{Effects of Eugenol on Oxygen Consumption Rate and Ammonia Excretion Rate of Aquatic Animals}

Generally, the oxygen consumption rate of aquatic animals anesthetized by eugenol anesthetic will rise first and then decrease. When the concentration increased further, the oxygen consumption rate of juvenile acipenserbaeri decreased gradually, and there were obvious differences occurring between different concentration [20]. When the concentration of eugenol was $10 \mathrm{mg} / \mathrm{L}$, the lowest oxygen consumption rate of trachinotusblochii was $0.33 \mathrm{mg} /(\mathrm{g} \mathrm{h})$ [21], and when the optimum concentration of eugenol for the carp anesthesia was $20 \mathrm{mg} / \mathrm{L}$, the oxygen consumption rate and ammonia excretion rate decreased significantly [22].

\subsection{Changes of biochemical parameters}

After luveniles of acipenserbaeriwere anesthetized with eugenol $60 \sim 180 \mathrm{mg} / \mathrm{L}$ for $10 \mathrm{~min}$., the concentrations of high-density lipoprotein, low density lipoprotein and triglyceride in the young group 
were significantly higher than those in the control group $(\mathrm{P}<0.05)$, while the total bilirubin concentration was lower than that in the control group $(\mathrm{P}<0.05)$; in the $90 \sim 180 \mathrm{mg} / \mathrm{L}$ concentration group, the magnesium concentration in the blood of the young fish was higher than that in the control group; in the $120 \sim 180 \mathrm{mg} / \mathrm{L}$ concentration group, the blood concentration of the young fish was higher than that of the control group. The concentration of glutamic oxaloacetic transaminase in the test group was higher than that in the control group. The concentration of albumin and lactic dehydrogenase in each test group fluctuated greatly. $8 \sim 30 \mathrm{mg} / \mathrm{L}$ eugenol could be applied to short-distance transportation of juvenile acipenserbaeri, and the influence of $60 \sim 90 \mathrm{mg} / \mathrm{L}$ eugenol on the blood biochemistry of juvenileacipenserbaeriwas relatively small.

\subsection{Tissue residues and safety of eugenol}

No carcinogenic or toxic effects of eugenol have been found in studies in various countries. The Standard for the Use of Food Additives GB2760-2014 specifies that natural plant extracts containing eugenol can be used as food additives [23]. However, there is no clear prescription for the use of eugenol as a fishery anesthetic. Moreover, the maximum residue limit of eugenol in food is not specified in Standard for the Use of Food Additives GB2760-2014 and Contaminant Limit in Food GB2762-2012[24]. Preliminary evaluation shows that eugenol used as a fish anesthetic has no effect on food safety.

Many countries have not approved the use of clove oil because it may contain methyl eugenol. In Japan and other countries, eugenol has been allowed to circulate in the market as a fish anesthetic. At present, there are no relevant regulations on the administration of eugenol used as fishery anesthetics in China. Some researchers suggest Chinese government that, for eugenol use management, we can refer to Japanese regulations on eugenol, and set temporary use and management limits. The specific dosage of medicine bath is $50 \sim 200 \mu \mathrm{g} / \mathrm{mL}$, and the residue limit is $0.05 \mu \mathrm{g} / \mathrm{mL}$. For the period of drug withdrawal, the dosage of fish is 7dayand that of crustaceans is 10day [25].

\section{SUMMARY}

With the development of the concept of "blue granary" in China, the development of fishery will reach an unprecedented speed and height. But if we want to develop modern fisheries, the standardization and use of anesthetics is an important step. China's research on fishery anesthetics started late, and there are many problems in the management and use of fishery anesthetics. (1)The relevant laws and regulations concerning the use of anesthetics in fisheries in China need to be improved and perfected, and there is a lack of allowed-to-use product catalog and using standards for the use of anesthetics for fisheries. (2)The pharmacology and metabolic pathways of anesthetic drugs in fish are relatively little studied. For many problems, such as intramuscular drug residues, safety range, toxicity and harm to human body, there is no credible basis for evaluation. (3)At present; there is a phenomenon of non-standard use of fishery anesthetics. To sum up, under the strategy of maritime power, it is imperative to solve the problems of fishery anesthetics if we want to develop the marine economy.

\section{ACKNOWLEDGMENT}

This research was financially supported by "Qing Lan Project of Jiangsu Province". This work was supported by "311 Talents Project in Taizhou" and Jiangsu Agri-animal Husbandry Vocational College (Grant No. NSF201711).

\section{REFERENCE}

[1] Zhang Longgang, Fu Pei Sheng,Yang Ling. Preliminary study on the anesthetic effect of MS-222 to the barbuscapito juvenile fish. Chinese Agricultural Science Bulletin, 2019(21).(In Chinese)

[2] Ding Yatao, Wang Zhihe, Wang Linlin and others. Study on anesthesia, preservation and transportation effects of MS-222 on bream. Aquatic Science,2019,38(03):296-304.(In Chinese)

[3] Lin Lizhen, Du Mingxi, Yu Lin Lin and so on. Study on the Anesthesia Effect of MS-222 on Pelteobagrusfulvidraco.Aquatic Science and Technology Information,2019,46(01):56-60.(In Chinese)

[4] Xu Bin, Wei Kaijin, Zhu Xiangyun and so on. Effects of MS-222 and eugenol on oxygen consumption rate, ammonia excretion rate and asphyxiation point of juvenile ProcambarusClarkii, Journal of Dalian Ocean University,2018,33(04):455-459.(In Chinese) 
[5] Jin Yuan, He Yajing, Cheng Bo, etc. Safety of eugenol anesthetics .Journal of Food Safety and Quality Detection, 2017,8(01):33-40.(In Chinese)

[6] Yan Yinlong, Shi Yonghai, Zhang Haiming, etc. Anesthetic effect of MS-222 and eugenol on juvenile coilia nasus.2016,25(02):177-182.(In Chinese)

[7] Liu Changlin, He Li, Chen Siqing, et al. A Review of Fish Anesthesia Research .Fisheries Modernization, 2007, 34(5):21-25.(In Chinese)

[8] Li Xiaoqin, Zhu Zhenhua, Zhai Jing Jing, etc. Research progress in the use and detection techniques of fishery anesthetics . Agricultural Sciences, 2017,45(29):72-73+170.(In Chinese)

[9] Dong Chun, Pan Lei and Qiao Ye. Overview of fishery anesthetics .Aquatic scientific and technological information, 2016,43(01):28-32+36.(In Chinese)

[10] Wang Lijuan, Cheng Shoukun, Zhang Yinjiang, etc. The anesthetic effect of MS-222 in simulated transport of bass .2015,24(02):235-241.(In Chinese)

[11] Tian Jiayuan, Wang Jingshu, Wan Jianyi, etc. Effects of different treatments of MS-222 on anesthetic effects of Acipenserschrenckii and Acipensersinensis.Journal of Aquatic Ecology,2011,32(05):87-90.(In Chinese)

[12] Lin Lizhen, Du Mingxi, Yu Lin Lin and so on. Study on the Anesthesia Effect of MS-222 on Pelteobagrusfulvidraco.Aquatic Science and Technology Information,2019, 46(01):62-66.(In Chinese)

[13] Yan Yinlong, Shi Yonghai, Zhang Haiming, etc. Anesthetic effect of MS-222 and eugenol on juvenile coilianasus . Journal of Shanghai Ocean University, 2016, 25(2):177-182.(In Chinese)

[14] Sun Xiaowang, Chen Yongping, Li Tong and so on. Study on the Anesthesia Effect and Elimination Law of MS-222 on cynoglossussemilaevis.Aquatic Science and Technology Information,2017,44(02):87-90.(In Chinese)

[15] Zhang Jiandong, Chen Gang, Tang Baogui and so on. Effects of anaesthetic MS222 on oxygen consumption and ammonia excretion rate of juvenile Nile tilapia.Fisheries modernization,2010,37(04):35-39.

[16] Xue Y J, Chang C C, Lai J M, et al. Determining the tranquilization dose and residue of tricainemethanesulfonate (MS-222) in sea bass Latescalcarifer tissue. Fisheries Science,2017,83(4):625-633.

[17] Popovic N T, Strunjak-Perovic I, Coz-Rakovac R, et al. Tricaine methane- sulfonate ( MS -222) application in fish anaesthesia. Journal of Applied Ichthyology,2012, 28(4):553-564.

[18] Bao Jie, Jiang Hongbo, Lu Huanyun and so on. Study on the anesthetic effect of clove oil on Loach (.)2014,45(05):621-624.(In Chinese)

[19] KuangGangqiao, Li Ping, Zheng Shuming, et al. Anesthetic effect of eugenol on young ictaluruspunctatus .Sichuan Journal of Zoology. 2010, 29(5):584-587.(In Chinese)

[20] Feng Guangpeng, Zhuangping, Zhang Longzhen, etc. Effects of anesthetic eugenol on oxygen consumption rate of juvenile acipenserbaeri and blood biochemical parameters . Journal of Dalian Fisheries University.2010,25(02):113-118.(In Chinese)

[21] Zhang Chaohui, Cong Jiaori, Wang Bo, etc. The effect of anesthetic eugenol on oxygen consumption of Trachinotusblochii .Oceanography,2003(06):11-14+34.(In Chinese)

[22] Zhang Fulin. Study on anesthetic effect of eugenol on carp [D]. Guizhou University,2008.(In Chinese)

[23] GB 2760-2011National food safety standard-Standard for uses of food additives [S].(In Chinese)

[24] GB2762-2012National food safety standard-Limits of pollutants in food [S].(In Chinese)

[25] SuMingming, Sun Xingquan, Yang Chunguang, etc. The application of fishery anesthetics MS-222 and eugenol in the transportation of fresh aquatic products and the progress of detection methods.Journal of Food Safety Quality Detection,2015,6(01): 25-29.(In Chinese)

Citation: Xiao-jiang Chen, "Research on the Application of Fishery Anesthetic MS-222 and Eugenol in China". International Journal of Innovative Studies in Aquatic Biology and Fisheries, 5(4), pp.1620.http://dx.doi.org/10.20431/2454-7670.0504004

Copyright: () 2019 Authors. This is an open-access article distributed under the terms of the Creative Commons Attribution License, which permits unrestricted use, distribution, and reproduction in any medium, provided the original author and source are credited. 